\title{
Multicriteria Handover Management by the SDN Controller-based Fussy AHP and VIKOR Methods
}

\author{
Najib Mouhassine ${ }^{1}$, Mohamed Moughit ${ }^{3}$ \\ LaSTI Laboratory, National School of Applied Sciences \\ Sultan Moulay Slimane University \\ Khouribga, Morocco
}

\author{
Mostapha Badri ${ }^{2}$ \\ MASI Laboratory,Polydisciplinary Faculty of Nador \\ Mohammed first university \\ Oujda,Morocco
}

\begin{abstract}
A wireless environment is characterized by its dynamic nature, inherent uncertainty, and imprecise parameters and constraints. Network settings such as speed, RSS, network delays, etc. are inherently imprecise. Due to this vagueness, accurately measuring these network parameters in a wireless environment is a difficult task. As a result, a fuzzy logic approach appears to work best when used to design systems in such environments. Although conventional techniques based on precise values can be used to reduce transmission delay, they cannot produce intelligent and efficient transfer decisions that take into account all the constraints of the network. Thus, using one criterion only can lead to service disruption, unbalanced network load, and inefficient handoff. Therefore, to guide the Horizontal handover process in wireless networks towards making a better choice for VoIP in congested environments, we propose the integration of the Fuzzy-AHP and VIKOR method in SDN (Software Defined Networking) controller on several criteria (the signal-to-noise ratio plus interference (SNIR), packet loss, jitter, delay, throughput). However, the results of this work show that our contribution maintains a good quality of service for real-time applications.
\end{abstract}

Keywords-SDN; QoS; WLAN; Handover; F-AHP; VIKOR

\section{INTRODUCTION}

In an uncertain environment, multi-criteria decisionmaking methods (MADM) consist of constructing a Global Preference Relation (optimal choice) for a set of alternatives (set of choices) evaluated by several criteria.

Each decision-making problem has several alternatives that a decision-maker tries to filter or classify in a decisionmaking process, also the attributes are essentially the criteria used to evaluate the alternatives. These attributes are grouped into attributes of cost and other benefits or risk. For example, mobile communication with a less noisy channel is advantageous, while the number of subscribers has a cost.

Each attribute can have its unit. The normalization is necessary to eliminate the effect of the different units; it consists of transforming the values of the alternatives into a scale included in the interval [0 1]. To reflect the importance of the attributes, they are assigned a weighting factor either by the decision-maker or calculated with a mathematical method.

MADM Problems can be formalized in matrix or vector form, where the columns and rows present the attributes (criteria) and the alternatives (choices). The elements of the matrix present the evaluation of the performance of the alternative against the attribute. For example, in the advantage attribute group (less noisy channel) alternatives with a higher score are preferred. In contrast, the cost attribute group (number of subscribers) alternatives with a low score are optimal.

In this work the F-AHP method presents the importance of each criterion over the other with the elements of the pairwise comparison matrix. After fuzzification and defuzzification come to assign a weight to each criterion.

Subsequently builds the selection matrix with the VIKORE method where the columns denote the weights of the criteria and the rows the choices (access point). Then calculate a coefficient which is used to classify the access points in order of importance. The values of the matrix are collected from OpenFlow access points in direct connection with the SDN controller.

Based on this classification made by the SDN controller, a message containing the order of priority is sent to the mobile which requests the change of the access point. The latter proceeds to trigger the Handover towards the right choice. Hence, this work is an improvement to our contribution [1].

\section{BACKGROUND}

The mathematical methods of multicriteria analysis can be grouped into two approaches:

\section{A. The Scalar Methods}

- Simple Additive Weighting method (SAW) [2].

Consists of weighting the different criteria of the multicriteria decision problem with real numbers called weights which represent the importance of each criterion in the decision process. Once the importance of the different criteria is quantified, the method chooses the action that minimizes or maximizes the weighted sum of the criteria.

Mathematical formulation:

Starting data

m actions $A_{1}, A_{2}, A_{3} \ldots A_{m}$.

n criteria $\mathrm{C}_{1}, \mathrm{C}_{2}, \mathrm{C}_{3} \ldots \mathrm{C}_{\mathrm{n}}$.

Weight vector $\left(\mathrm{W}_{1}, \mathrm{~W}_{2}, \ldots, \mathrm{W}_{\mathrm{n}}\right)$ et $\mathrm{W}_{\mathrm{j}}>0$. 
$a_{i j}=U_{i j}\left(A_{i}\right)$, Quotient cardinal utility function. Represents the performance of each action on each of the criteria.

Data transformation:

1) Normalization of aij.

2) Normalization of the weights.

3) Implementation of the weighted sum method (1).

$R\left(a_{i}\right)=\sum_{j=1}^{n} w_{j} a_{i j} \forall i \in[1, m]$

Single criterion for any action i.

- Weighted Product Mode (WPM) [2].

Similar to SAW, also called exponent weighting, the main difference is that we will have a multiplication instead of the addition. It allows comparing alternatives against several criteria. This comparison is made by dividing the values of all criteria by one of them and then multiplying those ratios. Each ratio is raised to the exponent equivalent to the weight of the corresponding criterion.

Mathematical formulation (2):

$P\left(\frac{A_{k}}{A_{L}}\right)=\prod_{j=1}^{N}\left(\frac{a_{k j}}{a_{L j}}\right)^{w_{j}} \quad k, L=12,3, \ldots$

Where $\mathrm{N}$ represents the number of criteria.

$\mathbf{m}$ the number of actions (or alternatives).

$\mathbf{w j}$ the weight of criterion $\mathbf{j}$.

$\mathbf{A}_{\mathbf{k}}$ and $\mathbf{A}_{\mathbf{L}}$ two alternatives to compare.

$\mathbf{a}_{\mathbf{K j}}$ and $\mathbf{a}_{\mathbf{L j}}$ the weights of the alternative $A_{k}$ and $A_{L}$

Compared to criterion $\mathrm{j}$.

We can also calculate the overall weight of an alternative by the following formula (3):

$P\left(A_{k}\right)=\prod_{j=1}^{N}\left(a_{k j}\right)^{w_{j}} k=1,2, \ldots m$

- Method of Weighted Metrics (MWM).

Selects a vector of criteria that minimizes the distance to a reference solution $R=\left(R_{1}, \ldots, R_{k}\right)$. The vector $R$ is either the ideal point or the reference solution fixed by the decisionmaker according to its preferences.

- Analytic Hierarchy Process (AHP) [3].

Allows calculating an aggregate synthetic score based on a ranking and weighting of all the criteria considered in the decision. It consists of four stages:

1) Establish the hierarchy of criteria and alternatives.

2) Perform pairwise comparisons of the criteria, and estimate the weights of the criteria and the relative performance values of the alternatives concerning each criterion.
3) Aggregate the weights and performance values for the alternative priority.

4) Check the consistency of judgments to verify the result The limitation of this method is the instability of the classification of the different alternatives if the problem to be treated contains a large number of alternatives.

- Analytical Network Process (ANP) [4].

It is an extension of the AHP and allows the consideration of the interdependence among and between the levels of criteria and alternatives.

- Multi Attribute Utility Theory (M.A.U.T) [5].

Method developed in the late 1960s by Ralph Keeney and Howard Raiffa attempting to modelize the preferences of the decision-maker by a so-called utility function using different mathematical tools. These methods are often of the aggregation type, in the sense that the resulting utility function is often an aggregation of several "sub-functions," which can be the objective functions of each criterion or a combination of two or more of them.

- The Technique for the Order of Preference by Similarity of Ideal Solution (TOPSIS) [6].

This technique proposed by [Yoon and Hwang, 1981] allows choosing the best solution which maximizes the profit criteria and minimizes the cost criteria. Firstly, it aims to reduce the number of disambiguation scenarios by discarding the dominated scenarios and, secondly, to rank the effective scenarios according to their calculated overall scores.

TOPSIS can sometimes be used to replace AHP in the process of ranking alternatives. In other words, it often happens that the AHP is used to assign the weight of the selection criteria while the TOPSIS is applied to prioritize the selection alternatives.

\section{B. Ranking Methods}

- Elimination And Choice Translating Reality (ELECTRE I) [7].

This method of Bernard Roy, allows a partial aggregation through the construction of comparison relations of the performances for each pair of solutions.

- Preference Ranking Organization METHod for Enrichment Evaluations (PROMETHEE) [8].

Consists of establishing a process of numerical comparison of each action in relation to all the other actions. Thus it is possible to calculate the plus or minus optimal of each action compared to all the others. The result of this comparison allows the orderly classification of the actions.

- VIKOR (VIsekriterijumska optimizacija i KOmpromisno Resenje) [9].

Multicriteria optimization and compromise solution: It is a ranking method for a finite set of alternatives and allows solving a discrete multicriteria problem with noncommensurable (different units) and contradictory criteria. 


\section{RELATED WORK}

In the context of our research, we can separate the work due to improving the Handover in three parts:

\section{A. Multi-Criteria or Multi-Attribute Decision-Making Algorithms (MCDM)}

R.Bikmukhamedov et al [10] evaluated the performances of SAW, GRA (Gray relational analysis) [11], TOPSIS and VIKOR algorithms, and they showed by simulations for the same criteria (RSSI, Available Bitrate and Cost) that VIKOR and GRA are optimal.

The VIKOR method was developed under the name Dynamic VIKOR (D-VIKOR) by Xiaohong Li et al [12], it reduces time complexity by frequently evaluating dynamic attributes. This helps to minimize the Handover delay, otherwise the execution time.

K. savita et al [2], carried out a comparative study between the two methods WPM and SAW for the attributes: delay, jitter and cost, to choose the best wireless network that offers minimal transmission delay. Without results, the judgment postulated on the performance of the WPM method remains useless.

M.Alhabo et al [13], propose two modified TOPSIS methods to reduce the number of unnecessary handovers and radio link failure, plus improving the average throughput for each user. One integrates the entropy weighting of metrics (PE-TOPSIS), and the other integrates the weighting of the standard deviation (PSD-TOPSIS) to note the importance of each criterion (here the angle of movement, time of stay, the signal-to-noise ratio plus interference). The simulation is done with a constant mobility speed and random deviation angles. The results are evaluated in terms of the number of handovers, radio link failures, and average user throughput compared to the two network-controlled methods Handover and TOPSIS.

Another implementation of TOPSIS was carried out by R.Abdullah and Z.Zukarnain [14], to select the most relevant wireless access technology (LTE, WiMAX, WLAN). Three types of priority are defined, namely equal priority, Mobile priority and Network priority. The simulation results prove that the network priority is better than the two other.

\section{B. The Fuzzy Logic Theory}

Network parameters such as delay, jitter, noise, effective throughput, etc. are generally imprecise and unpredictable, measurements are approximate and probabilistic. Therefore, fuzzy logic allows us to model this imprecision for each parameter by linguistic variables, that each variable corresponds to a membership function having a value in the interval $[0,1]$.

Implementation of fuzzy logic made by A.Sadik et al. [15], aims to choose the best AP (access point) and reduce the handover delay. They chose the RSSI and the direction from the mobile node to the AP as selection criteria.

The classification of the network for the desired application according to user preferences and also network parameters has been the research objectives of M. Krichna et al. [16]. The chosen method uses the concept of fuzzy logic in three steps, the first is the initiation phase of the Handover based on two parameters, QoS and the power of the signal (RSS), which are the inputs of FIS (Fuzzy Inference System) mamdani, the second is the preselecting phase which takes into account the speed of mobility and user preferences, finally the classification of WLAN, WIMAX and cellular networks based on the type of application chosen.

M.sharma [17], had also introduced fuzzy logic (criterion: RSS, Bandwidth, Users Preference) to trigger the Handover towards choosing the optimal network between WLAN and WWAN.

\section{Fuzzy Logic Combined with MCDM Algorithms}

In heterogeneous wireless networks, mobile equipment is equipped with various wireless access technologies, to allow it to intelligently choose the one that offers the best quality of communication. F.kaleem et al. [18], has just proposed a combined technique in two steps. The first consists of estimating the need to trigger the Handover towards another technology based on the fuzzy multicriteria decision. The second is the selection of a target PoA (Point-of-Attachment) based on ranking algorithms, namely TOPSIS with AHP weighting, TOPSIS with F-AHP (Fuzzy AHP) weighting and FTOPSIS (fuzzy TOPSIS). The fuzzy system input parameters are, predicted RSS, mobility speed, the distance between mobile stations and PoA, and degree of QoS. the output of the system is the Vertical Handover Factor. The degree of QoS is calculated according to the class traffic type requirements currently used. According to the network parameters, the weights assigned to each class are calculated with the AHP or Fuzzy AHP method (throughput, jitter, delay, and packet loss rate).

M.Alkhawlani et al. [19], implemented a decision-making system based on the combination of fuzzy logic and TOPSIS, for the choice between three types of networks.

To remove the ambiguity, the criteria present the input of the fuzzy logic system. At the same time, the outputs of the latter, are the inputs of the MCDM TOPSIS system, which allows after construction of the decision matrix, weighting and determination of the best and worst value for each attribute by the max-min-distance method, to select the network that offers an optimal solution. The results are compared to each criterion with the proposed system.

S. Zhang et al. [20], had suggested the Fuzzy GRA method for solving multicriteria problems. Fuzzy numbers are defined in triangular value intervals, criteria weighting and selection are calculated by the GRA method.

In most research, the criteria weighting step is done with the AHP method or its Fuzzy-AHP Enhanced Version to determine the importance of each attribute. Drissi et al [21], evaluated the performance of the F-AHP method compared to AHP for different decision algorithms (MEW, SAW, VIKOR, TOPSIS) and different types of traffic. The results show better QoS by combining F-AHP with the VIKOR method.

\section{Problem Statement and Proposed Model}

According to the comparison of previous research focused on maintaining a good quality of service in wireless networks, 
the rest of this article proposes the management of multicriteria handover by the SDN controller based on the FAHP and VIKOR. The problem can be formalized as described in "Fig. 1", where each access point calculates its parameters and sends them to the controller, which ranks them in order of QoS priority.

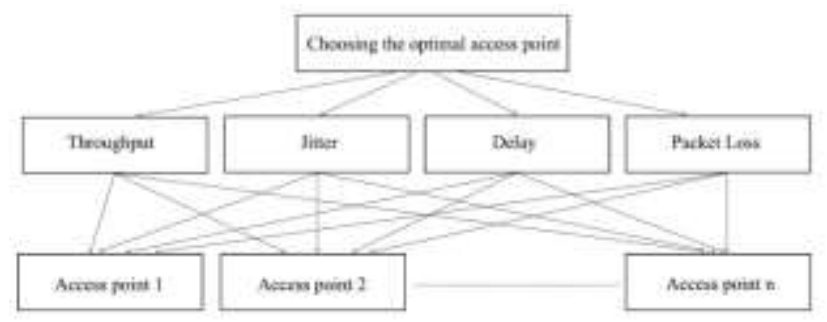

Fig. 1. The Graphical Formalism of the Problem.

The solution of the problem is divided into two stages:

\section{A. Weighting of Criteria by the F-AHP Method}

1) Creation of the pairwise comparison matrix "Table I": The weighting vectors give more importance to the delays then the jitter and finally the loss of the packets, the throughput in the case of VoIP is not so important because the packets are small and can be transmitted at a low rate.

2) Fuzzification "Table II": creation of the fuzzy AHP pairwise comparison matrix.

The fuzzy geometric mean value "Table III" is calculated by this formula (4) (Buckley 1985) [22].

$r_{i}=\left(\prod_{j=1}^{n} a_{i j}\right)^{1 / n}$

Product of two fuzzy numbers.

$\widetilde{\mathrm{A}}_{1} \otimes \widetilde{\mathrm{A}}_{2}=\left(\mathrm{l}_{1}, \mathrm{~m}_{1}, \mathrm{U}_{1}\right) \otimes\left(\mathrm{L}_{2}, \mathrm{~m}_{2}, \mathrm{U}_{2}\right)$

$=\left(\mathrm{l}_{1} * \mathrm{l}_{2}, \mathrm{~m}_{1} * \mathrm{~m}_{2}, \mathrm{U}_{1} * \mathrm{U}_{2}\right)$

Criteria matrix $\overline{\mathrm{A}}=\left[\mathrm{a}_{\mathrm{ij}}\right]$

TABLE I. PAIRWISE COMPARISON MATRIX

\begin{tabular}{|l|l|l|l|l|}
\hline Conversation & Packet Loss & Delay & Jitter & Throughput \\
\hline Packet Loss & 1 & $1 / 7$ & $1 / 5$ & 3 \\
\hline Delay & 7 & 1 & $1 / 3$ & 7 \\
\hline Jitter & 5 & 3 & 1 & 7 \\
\hline Throughput & $1 / 3$ & $1 / 7$ & $1 / 7$ & 1 \\
\hline
\end{tabular}

TABLE II. FUZZY AHP PAIRWISE COMPARISON MATRIX

\begin{tabular}{|l|l|l|l|l|}
\hline Conversation & $\begin{array}{l}\text { Packet } \\
\text { Loss }\end{array}$ & Delay & Jitter & Throughput \\
\hline Packet Loss & $(1,1,1)$ & $(1 / 8,1 / 7,1 / 6)$ & $(1 / 6,1 / 5,1 / 4)$ & $(2,3,4)$ \\
\hline Delay & $(6,7,8)$ & $(1,1,1)$ & $(1 / 4,1 / 3,1 / 2)$ & $(6,7,8)$ \\
\hline Jitter & $(4,5,6)$ & $(2,3,4)$ & $(1,1,1)$ & $(6,7,8)$ \\
\hline Throughput & $(1 / 4,1 / 3,1 / 2)$ & $(1 / 8,1 / 7,1 / 6)$ & $(1 / 8,1 / 7,1 / 6)$ & $(1,1,1)$ \\
\hline
\end{tabular}

TABLE III. FUZZY GEOMETRIC MEAN VALUE RI

\begin{tabular}{|l|l|}
\hline Conversation & $\boldsymbol{r}_{\boldsymbol{i}}$ value \\
\hline Packet Loss & $(0.45,0.54,0.64)$ \\
\hline Delay & $(1.73,2.01,2.38)$ \\
\hline Jitter & $(2.63,3.20,3.72)$ \\
\hline Throughput & $(0.70,0.29,0.34)$ \\
\hline
\end{tabular}

This formula (5) calculates the fuzzy weights for each criterion "Table IV".

$\mathrm{W}_{\mathrm{i}}=\mathrm{r}_{\mathrm{i}} \otimes\left(\mathrm{r}_{1} \oplus \mathrm{r}_{2} \oplus \ldots \oplus \mathrm{r}_{\mathrm{n}}\right)^{-1}$

Sum of two fuzzy numbers.

$\widetilde{\mathrm{A}}_{1} \oplus \widetilde{\mathrm{A}}_{2}=\left(\mathrm{l}_{1}, \mathrm{~m}_{1}, \mathrm{U}_{1}\right) \oplus\left(\mathrm{L}_{2}, \mathrm{~m}_{2}, \mathrm{U}_{2}\right)$

$=\left(\mathrm{l}_{1}+\mathrm{l}_{2}, \mathrm{~m}_{1}+\mathrm{m}_{2}, \mathrm{U}_{1}+\mathrm{U}_{2}\right)$

So

$\left(\mathrm{r}_{1} \oplus \mathrm{r}_{2} \oplus \ldots \oplus \mathrm{r}_{\mathrm{n}}\right)=(0.45+1.73+2.63+0.70,0.54+$ $2.01+3.20+0.29,0.64+2.38+3.72+0.34)$

$=(5.51,6.04,7.08)$

$\left(r_{1} \oplus r_{2} \oplus \ldots \oplus r_{n}\right)^{-1}=\left(\frac{1}{7.08}, \frac{1}{6.04}, \frac{1}{5.51}\right)$

TABLE IV. FUZZY WEIGHTS FOR EACH CRITERIA

\begin{tabular}{|l|l|l|}
\hline & Fuzzy geometric mean value $\boldsymbol{r}_{\boldsymbol{i}}$ & Fuzzy weights $\boldsymbol{W}_{\boldsymbol{i}}$ \\
\hline Packet Loss & $(0.45,0.54,0.64)$ & $(0.064,0.089,0.12)$ \\
\hline Delay & $(1.73,2.01,2.38)$ & $(0.244,0.332,0.431)$ \\
\hline Jitter & $(2.63,3.20,3.72)$ & $(0.371,0.530,0.675)$ \\
\hline Throughput & $(0.70,0.29,0.34)$ & $(0.099,0.048,0.062)$ \\
\hline
\end{tabular}

3) Defuzzification to get crisp numerical values "Table V": The defuzzification method used here is center of area (CoA) (6).

$w_{i}=\left(\frac{l+m+u}{3}\right)$

TABLE V. CRISP NUMERICAL VALUES

\begin{tabular}{|l|l|c|}
\hline & Weight $\boldsymbol{w}_{\boldsymbol{i}}$ & Normalised weight \\
\hline Packet Loss & 0.137 & $\frac{0.137}{1.068}=0,128$ \\
\hline Delay & 0.336 & $\frac{0.336}{1.068}=0,315$ \\
\hline Jitter & 0.525 & $\frac{0.525}{1.068}=0,492$ \\
\hline Throughput & 0.070 & $\frac{0.070}{1.068}=0,065$ \\
\hline Sum & $1.068>1$ & 1 \\
\hline
\end{tabular}

B. Access Points Classification by VIKOR Method

1st step: find the best " $x_{i}^{+}$" (7) and the worst " $x_{i}^{-}$" (8) value for each criteria $\mathrm{j}=1,2, \ldots, \mathrm{n}$ "Table VI". 
$x_{i}^{+}=\left\{\left(\max \left(\mathrm{n}_{\mathrm{ij}}\right) \mid \mathrm{j} \subset \mathrm{M}_{\mathrm{p}}\right) ;\left\{\left(\min \left(\mathrm{n}_{\mathrm{ij}}\right) \mid \mathrm{j} \subset \mathrm{M}_{\mathrm{c}}\right)\right\}\right.$
$\mathrm{i}=1,2, \cdots \mathrm{m}$

$\mathrm{x}_{\mathrm{i}}^{-}=\left\{\begin{array}{c}\left\{\left(\min \left(\mathrm{n}_{\mathrm{ij}}\right) \mid \mathrm{j} \subset \mathrm{M}_{\mathrm{p}}\right) ;\left\{\left(\max \left(\mathrm{n}_{\mathrm{ij}}\right) \mid \mathrm{j} \subset \mathrm{M}_{\mathrm{c}}\right)\right\}\right. \\ \mathrm{i}=1,2, \cdots \mathrm{m}\end{array}\right.$

With $\mathrm{i}=1,2, \cdots \mathrm{m}$ presents the alternatives (Access point).

Where $\mathrm{Mp}$ and $\mathrm{Mc}$ are the sets of performance and cost parameters, respectively.

2nd step: compute $S_{i}(9)$ and $R_{i}(10)$ value for each access point "Table VII".

$S_{i}=\sum_{j=1}^{n} w_{j} \cdot\left(\frac{x_{i}^{+}-x_{i j}}{x_{i}^{+}-x_{i}^{-}}\right)$

$R_{i}=\max _{j}\left[w_{j} \cdot\left(\frac{x_{i}^{+}-x_{i j}}{x_{i}^{+}-x_{i}^{-}}\right)\right]$

"Table VIII" shows the values $\left(S^{*}, R^{*}\right)$ and $\left(S^{-}, R^{-}\right)$ which respectively present the minimum and maximum value of $\mathrm{Si}$ and $\mathrm{Ri}$ for each access point.

$\mathrm{S}^{*}=\min _{\mathrm{i}} \mathrm{S}_{\mathrm{i}} ; \mathrm{R}^{*}=\min _{\mathrm{i}} \mathrm{R}_{\mathrm{i}}$;

$\mathrm{S}^{-}=\max _{\mathrm{i}} \mathrm{S}_{\mathrm{i}} ; \mathrm{R}^{-}=\max _{\mathrm{i}} \mathrm{R}_{\mathrm{i}}$.

3rd step: compute $Q_{i}$ value (11) "Table IX".

$Q_{i}=v \cdot \frac{S_{i}-S^{*}}{S^{-}-S^{*}}+(1-v) \cdot \frac{R_{i}-R^{*}}{R^{-}-R^{*}}$

where $v=0,5$.

TABLE VI. BEST AND THE WORST VALUE FOR EACH CRITERION

\begin{tabular}{|l|l|l|l|l|}
\hline Weight & \multicolumn{1}{|c|}{0,128} & \multicolumn{1}{|c|}{0,315} & \multicolumn{1}{|c|}{0,492} & \multicolumn{1}{c|}{0,065} \\
\hline Criteria & Packet Loss & Delay & Jitter & Throughput \\
\hline AP1 & 0.01 & $150 \mathrm{~ms}$ & $30 \mathrm{~ms}$ & $20 \mathrm{Mb} / \mathrm{s}$ \\
\hline AP2 & 0.03 & $100 \mathrm{~ms}$ & $15 \mathrm{~ms}$ & $15 \mathrm{Mb} / \mathrm{s}$ \\
\hline AP3 & 0.02 & $250 \mathrm{~ms}$ & $20 \mathrm{~ms}$ & $10 \mathrm{Mb} / \mathrm{s}$ \\
\hline Best $\left(\boldsymbol{x}_{\boldsymbol{i}}^{+}\right)$ & 0.01 & 100 & 15 & 20 \\
\hline Worst $\left(\boldsymbol{x}_{\boldsymbol{i}}^{-}\right)$ & 0.03 & 250 & 30 & 10 \\
\hline
\end{tabular}

TABLE VII. $S_{i}$ AND $R_{i}$ VALUE FOR EACH ACCESS PoINT

\begin{tabular}{|l|l|l|l|l|l|l|}
\hline Weight & 0,128 & 0,315 & 0,492 & 0,065 & $S_{i}$ & $R_{i}$ \\
\hline $\mathbf{A P}_{\mathbf{1}}$ & 0 & 0,105 & 0,492 & 0 & 0,597 & 0,492 \\
\hline $\mathbf{A P}_{\mathbf{2}}$ & 0,128 & 0 & 0 & 0,0325 & 0,1605 & 0,128 \\
\hline $\mathbf{A P}_{\mathbf{3}}$ & 0,064 & 0,315 & 0,164 & 0,065 & 0,608 & 0,315 \\
\hline
\end{tabular}

TABLE VIII. ( $\left.\mathrm{S}^{*}, \mathrm{R}^{*}\right)$ AND $\left(S^{-}, R^{-}\right)$VALUE FOR EACH ACCESS POINT

\begin{tabular}{|l|l|c|}
\hline & \multicolumn{1}{|c|}{$\boldsymbol{S}_{\boldsymbol{i}}$} & $\boldsymbol{R}_{\boldsymbol{i}}$ \\
\hline $\mathbf{A P}_{\mathbf{1}}$ & 0,597 & 0,492 \\
\hline $\mathbf{A P}_{\mathbf{2}}$ & 0,1605 & 0,128 \\
\hline $\mathbf{A} \mathbf{P}_{\mathbf{3}}$ & 0,608 & 0,315 \\
\hline $\boldsymbol{S}^{*}, \boldsymbol{R}^{*}$ & 0,1605 & 0,128 \\
\hline $\boldsymbol{S}^{-}, \boldsymbol{R}^{-}$ & 0,608 & 0,492 \\
\hline
\end{tabular}

TABLE IX. COMPUTE Qi VALUE

\begin{tabular}{|l|l|c|c|l|}
\hline & \multicolumn{1}{|c|}{$\boldsymbol{S}_{\boldsymbol{i}}$} & $\boldsymbol{R}_{\boldsymbol{i}}$ & $\boldsymbol{Q}_{\boldsymbol{i}}$ & Rank base on $\boldsymbol{Q}_{\boldsymbol{i}}$ \\
\hline $\mathbf{A P}_{\mathbf{1}}$ & 0,597 & 0,492 & 0,988 & 3 \\
\hline $\mathbf{A P}_{2}$ & 0,1605 & 0,128 & 0 & 1 \\
\hline $\mathbf{A P}_{3}$ & 0,608 & 0,315 & 0,756 & 2 \\
\hline $\boldsymbol{S}^{*}, \boldsymbol{R}^{*}$ & 0,1605 & 0,128 & & \\
\hline $\boldsymbol{S}^{-}, \boldsymbol{R}^{-}$ & 0,608 & 0,492 & & \\
\hline
\end{tabular}

Based on the calculated Qi values, the access points are ranked in order of QoS priority.

\section{IMPLEMENTATION, SIMULATION AND RESULTS}

Fig. 2 shows the algorithm implemented to manage Handover. The SDN controller periodically collects every 5 seconds the information from the access points namely jitter, delay, packet loss and Throughput, on which it calculates the order of QoS priority for each access point. A mobile that sees a SNIR value below the threshold sends a request to the controller, which responds with the list of access points already classified. The mobile connects to the access point which offers a good quality of service.

To evaluate our contribution, we tried to make a comparative study of two codecs G711 and G729, for a network architecture with SDN and other SDN based on FAHP and VIKOR methods. The following "Table X" shows some characteristics of the two codecs.

TABLE X. CODECS CHARACTERISTICS

\begin{tabular}{|l|l|l|l|l|}
\hline Codec & $\begin{array}{l}\text { Bit Rate } \\
(\text { Kbit/s) }\end{array}$ & $\begin{array}{l}\text { Link Utilization } \\
(\text { Kbit/s) }\end{array}$ & Delay (ms) & Loss $(\%)$ \\
\hline G.711 & 64 & 87.2 & 0.125 & $7-10$ \\
\hline G.729 & 8.0 & 31.2 & 15 & $<2$ \\
\hline
\end{tabular}

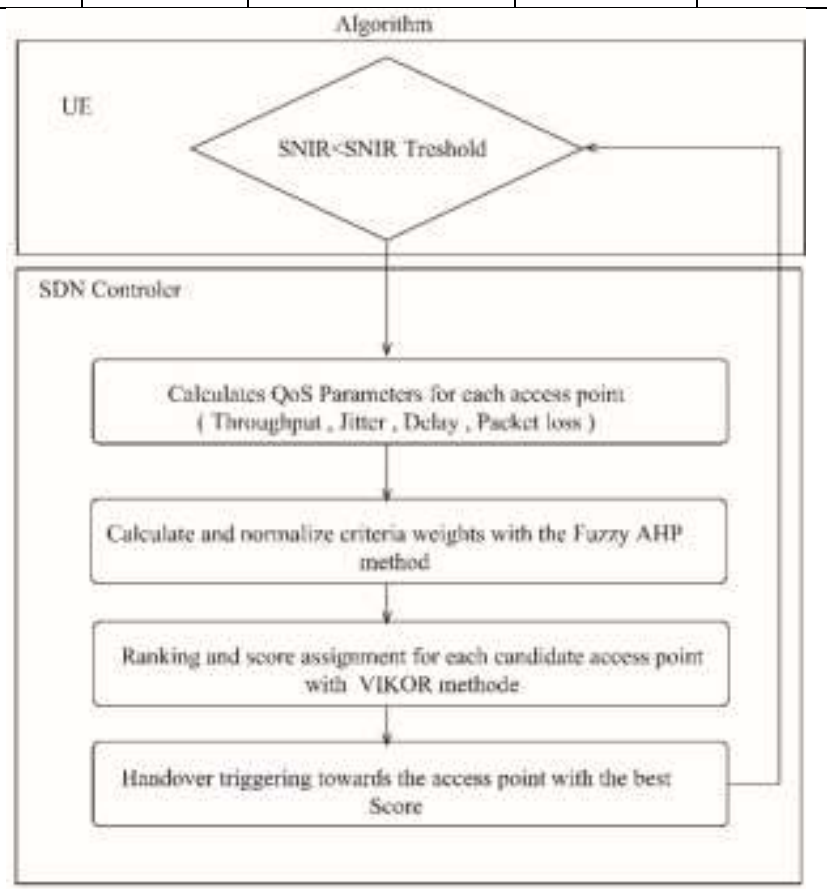

Fig. 2. The Proposed Model Flowchart. 
Also, the simulation architecture "Fig. 3" is composed of three APs (access point) that support the OpenFlow v1.3 protocol; thus, an SDN controller and a VoIPStreaming server. More than 20 nodes follow linear mobility with variable speed, and communicate progressively over time with the server. The simulator chosen for this experiment is OMNeT ++ 5.5.1.

Our controller is configured to perform both functions, classification of access points according to QoS priority and switching. The simulations are repeated ten times per experiment, and the results illustrated below present their averages.

\section{A. End-to-end Delay}

The end-to-end delay represents the propagation delay plus the delay introduced by the equipment, also the coding, decoding and packing time.

The mobile no longer makes a blind choice without prior knowledge of the quality of service offered by the access points, the decision calculated and taken by the controller based on information collected periodically from them, then sent on request to the mobile. So, the time that will commit the motive for the calculation of the decision before the execution of the Handover is reduced. Also, the delay in processing packets by the access points is minimized. The controller is responsible for instructing the access points on how to route the packets to the recipient. This explains the results obtained "Fig. 4", where the end-to-end delay was significantly reduced for both codecs by implementing our solution. In the case of the G729, the delay no longer exceeds $100 \mathrm{~ms}$. While for G711 is limited to $200 \mathrm{~ms}$.

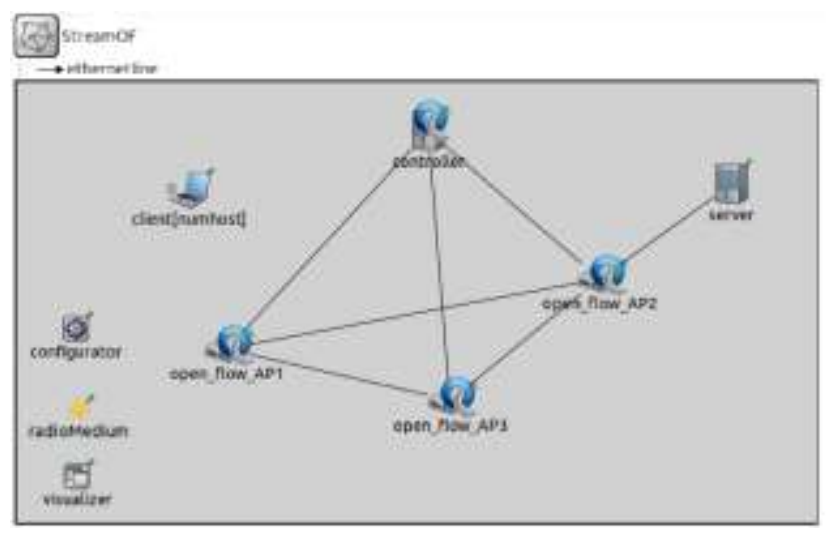

Fig. 3. Simulation Architecture.

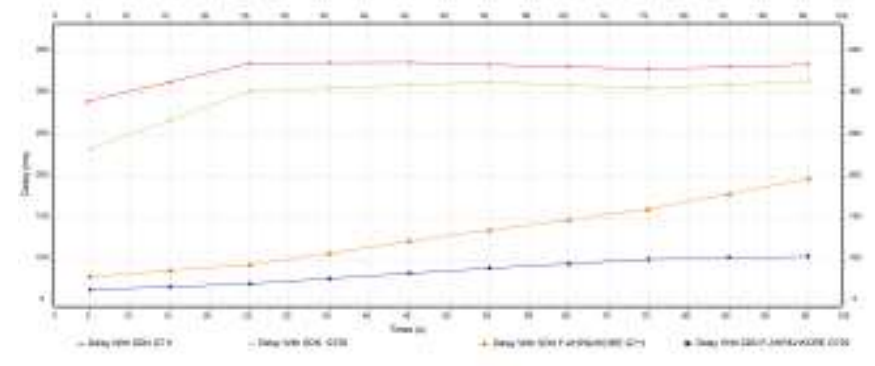

Fig. 4. Delay Comparison between SDN and SDN based F-AHP \& VIKOR.

\section{B. Jitter}

It refers to the difference in end-to-end transmission delay between different packets of the same stream when transmitting from one system to another; hence, the reason for the results presented in the following figure "Fig. 5". The SDN controller, which incorporates the multicriteria choice technique based on F-AHP and VIKORE produced good jitter values no longer exceeding $18 \mathrm{~ms}$ for the G711 codec, also note that the G729 codec is still more powerful than G711 and has values no longer exceeding $13 \mathrm{~ms}$.

\section{Packet Loss}

Packet loss occurs when one or more data packets passing through a computer network cannot reach their destination. Usually in wireless networks, it is caused by data transmission errors, interference, very low radio power, or network congestion. The following formula (12) gives the percentage value of lost packets:

PL $\%=\frac{\text { packets sent }- \text { packets received }}{\text { packets sent }}$

According to the results shown in "Fig. 6", the G711 codec maintains its packet loss resistance with SDN technology based on multicriteria choice, it does not cross the $1 \%$ limits. Even for G729 in the worst cases, reached an acceptable value below $2 \%$.

\section{Mean Opinion Score (MOS)}

Model E (ITU G.107) [23] provides a powerful means of evaluating and predicting the quality of calls in data networks. The calculated result of model $\mathrm{E}$ is a single scalar, called "Rvalue", which is derived based on the delay and degradation factors of the equipment. Once the $\mathrm{R}$ value is obtained, it can be mapped to the estimated MOS, it ranges from 100 to 0 , where 100 is excellent and 0 is poor. When the signal is not altered, the quality is perfect, we write (13):

$\mathrm{R}=\mathrm{R} 0$

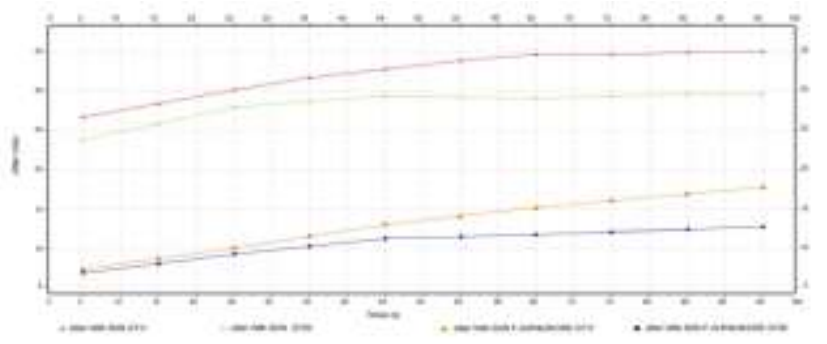

Fig. 5. Jitter Comparison between SDN and SDN based F-AHP \& VIKOR.

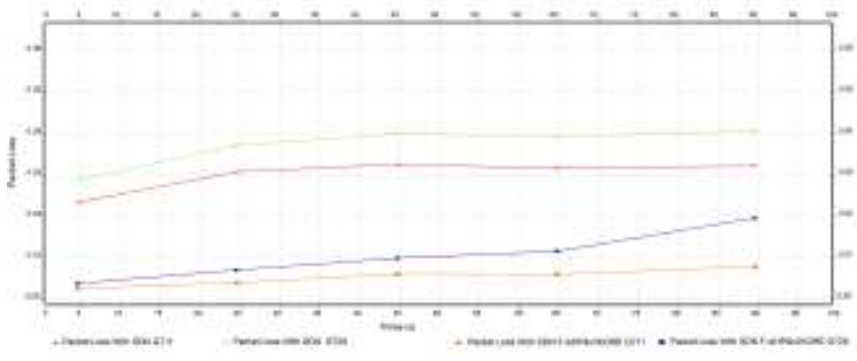

Fig. 6. Packet Loss Comparison between SDN and SDN based F-AHP \& VIKOR. 
In reality, the signal is influenced by the equipment and the constraints of the network, which degrades its quality from end to end (14).

$\mathrm{R}=\mathrm{R} 0-\mathrm{Id}-\mathrm{Ie}+\mathrm{A}$

With:

- Id is the end-to-end delay.

- Ie impairment introduced by the equipment.

- R0 Basic signal-to-noise ratio.

- A Advantage factor.

These values are related to data loss, jitter and delay, also the chosen codec. The choice of codec is a compromise between the desired quality of service and the ability of the IP infrastructure to deliver bandwidth and QoS parameters that will impact this quality. For example, the G711 codec offers a better quality of the channel since it does not perform any compression, therefore less delay and less sensitive to datagram losses. While other codecs like G729 and G723 consume less bandwidth, allowing simultaneous calls to be made on the same segment, but introduce more delay due to compression. These are sensitive to datagram loss. SDN technology provides fair quality for the G729 codec and good for the G711 codec. Fig. 7 shows that the integration of our SDN solution based on F-AHP and VIKORE helps the mobile node to make a good decision and brought an improvement in audio quality.

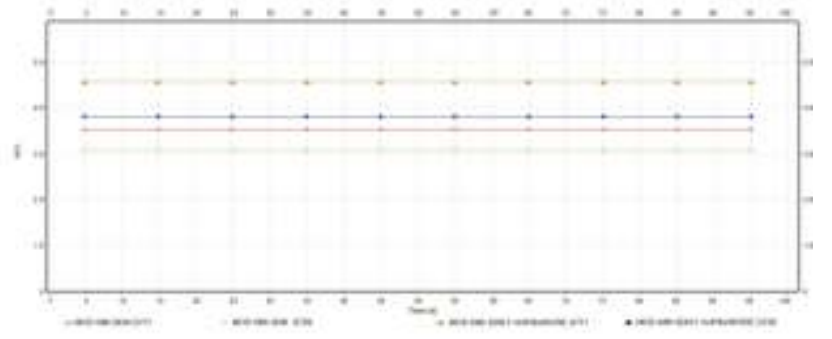

Fig. 7. MOS Comparison between SDN and SDN based F-AHP \& VIKOR.

\section{CONCLUSION}

SDN technology presents good quality in wired networks, contrary to a wireless architecture influenced by constraints of an unpredictable nature. To solve this problem, we tried to integrate the multi-criterion management of Handover by SDN controller based on F-AHP and VIKOR.

The results showed a good quality of service for real-time applications, since the multi-criterion choice is based on a weighting technique which takes into account the fuzzy nature of the network parameters and also the importance of each criterion in relation to each other depending on the type of traffic chosen.

The VIKOR classification method consumes less resources which generates flexibility in the decision, and decreases the time required to trigger the handover. The mobile node is in front of a safe choice, without leaning into computations that decrease battery power and also increase the handover trigger delay or packet loss.
For large networks, it is possible to envisage processing distributed by several SDN controllers based on F-AHP and VIKOR.

\section{REFERENCES}

[1] N. Mouhassine, M. Moughit, and F. Laassiri, "Improving the Quality of Service of Voice over IP in Wireless Sensor Networks by Centralizing Handover Management and Authentication Using the SDN Controller," 2019 3rd International Conference on Intelligent Computing in Data Sciences, ICDS 2019, 2019, doi: 10.1109/ICDS47004.2019.8942345.

[2] K. Savitha and Dr. C. Chandrasekar, "Vertical Handover decision schemes using SAW and WPM for Network selection in Heterogeneous Wireless Networks," Global Journal of Computer Science and Technology, vol. 11, no. 9, p. 7, 2011, doi: 10.5120/3125-4300.

[3] T. L. Saaty, "Transport planning with multiple criteria: The analytic hierarchy process applications and progress review," Journal of Advanced Transportation, vol. 29, no. 1, pp. 81-126, 1995, doi: 10.1002/atr.5670290109.

[4] The analytic network process, vol. 195. 2013.

[5] R. L. Keeney, H. Raiffa, and R. F. Meyer, Decisions with multiple objectives: preferences and value trade-offs. Cambridge university press, 1993.

[6] C.-L. Hwang and K. Yoon, "Methods for multiple attribute decision making," in Multiple attribute decision making, Springer, 1981, pp. 58191.

[7] BENADLA Abdelhalim, "Etude du Multihoming dans les Terminaux Mobiles (Sélection d'Interface)," pp. 2012-2013, 2013.

[8] J. P. Brans and B. Mareschal, "The PROMETHEE methods for MCDM; the PROMCALC, GAIA and BANKADVISER software," in Readings in multiple criteria decision aid, Springer, 1990, pp. 216-252.

[9] S. Opricovic and G. H. Tzeng, "Extended VIKOR method in comparison with outranking methods," European Journal of Operational Research, vol. 178, no. 2, pp. 514-529, 2007, doi: 10.1016/j.ejor.2006.01.020.

[10] R. Bikmukhamedov, A. Nadeev, Y. Yeryomin, and J. Seitz, "Simulating multi-criteria handover algorithms in OMNeT++," 2017 International Siberian Conference on Control and Communications, SIBCON 2017 Proceedings, 2017, doi: 10.1109/SIBCON.2017.7998466.

[11] Y. Kuo, T. Yang, and G.-W. Huang, "The use of grey relational analysis in solving multiple attribute decision-making problems," Computers \& industrial engineering, vol. 55, no. 1, pp. 80-93, 2008.

[12] X. Li, F. Liu, Z. Feng, G. Xu, and Z. Fu, "A novel optimized vertical handover framework for seamless networking integration in cyberenabled systems," Future Generation Computer Systems, vol. 79, pp. 417-430, 2018, doi: 10.1016/j.future.2017.03.031.

[13] M. Alhabo and L. Zhang, "Multi-criteria handover using modified weighted TOPSIS methods for heterogeneous networks," IEEE Access, vol. 6, no. April 2020, pp. 40547-40558, 2018, doi: 10.1109/ACCESS.2018.2846045.

[14] R. M. Abdullah and Z. A. Zukarnain, "Enhanced handover decision algorithm in heterogeneous wireless network," Sensors (Switzerland), vol. 17, no. 7, 2017, doi: 10.3390/s17071626.

[15] A. S. Sadiq, K. Abu Bakar, and K. Z. Ghafoor, "a Fuzzy Logic Approach for Reducing Handover Latency in Wireless Networks," Network Protocols and Algorithms, vol. 2, no. 4, pp. 61-87, 2011, doi: 10.5296/npa.v2i4.527.

[16] M. V. S. Krishna and L. Rajesh, "Implementation of fuzzy logic for network selection in next generation networks," International Conference on Recent Trends in Information Technology, ICRTIT 2011, pp. 595-600, 2011, doi: 10.1109/ICRTIT.2011.5972475.

[17] M. Sharma, "Fuzzy Logic Based Handover Decision System," International Journal of Ad hoc, Sensor \& Ubiquitous Computing, vol. 3, no. 4, pp. 21-29, 2012, doi: 10.5121/ijasuc.2012.3403.

[18] F. Kaleem, A. Mehbodniya, K. K. Yen, and F. Adachi, "A fuzzy preprocessing module for optimizing the access network selection in wireless networks," Advances in Fuzzy Systems, vol. 2013, 2013, doi: 10.1155/2013/232198. 
[19] M. M. Alkhawlani, K. A. Alsalem, and A. A. Hussein, "Multi-criteria vertical handover by TOPSIS and fuzzy logic," 2011 International Conference on Communications and Information Technology, ICCIT 2011, pp. 96-102, 2011, doi: 10.1109/ICCITECHNOL.2011.5762703.

[20] S. F. Zhang, S. Y. Liu, and R. H. Zhai, "An extended GRA method for MCDM with interval-valued triangular fuzzy assessments and unknown weights," Computers and Industrial Engineering, vol. 61, no. 4, pp. 1336-1341, 2011, doi: 10.1016/j.cie.2011.08.008.
[21] M. Drissi, M. Oumsis, and D. Aboutajdine, "A Multi-Criteria Decision Framework for network selection over LTE and WLAN," Engineering Applications of Artificial Intelligence, vol. 66, no. February, pp. 113127, 2017, doi: 10.1016/j.engappai.2017.08.019.

[22] J. J. Buckley, "Fuzzy hierarchical analysis," Fuzzy Sets and Systems, vol. 17, no. 3, pp. 233-247, 1985, doi: 10.1016/0165-0114(85)90090-9.

[23] D. Systems, “ITU-T,” 2015. 